\title{
Adidas. El logotipo como discurso visual. Definición de un modelo de análisis y estudio de caso
}

Óliver Pérez Latorre I oliver.perez@upf.edu Universitat Pompeu FABRA

Resumen: Este artículo tiene como objetivo la definición de un modelo de análisis para el estudio de los logotipos como discurso. Al margen de su función de "marcaje" comercial, el logotipo condensa los valores de la compañía constituyendo un núcleo de significación de la misma, crea valor diferencial respecto a compañías de la competencia y, con el tiempo, puede pasar a formar parte de la cultura popular. Este trabajo plantea la fundamentación teórica de un modelo de análisis para el estudio de la imagen-logotipo como discurso visual, basado en el Análisis Semiótico del Discurso, la Semiótica de la Imagen y la Psicología de la Percepción Visual. El modelo de análisis propuesto se pone a prueba a través de un estudio de caso: el análisis del logotipo de Adidas como discurso.

Palabras clave: Logotipo, imagen, análisis del discurso, semiótica

Abstract: The aim of this paper is the definition of a model of analysis of logotypes as discourse. Besides its function as a commercial mark, the logotype condenses the values of the company and constitutes its core of signification. In addition, the discourse of logotypes makes a difference with other companies and in the end logotypes can become part of popular culture. This paper provides the theoretical fundamentals of a model of analysis for the study of logotypes as visual discourses. The theoretical framework of the research is built upon Semiotic Analysis of Discourse, Visual Semiotics and the Psychology of Visual Perception. The proposed model is tested in a case study: the analysis of the Adidas' logotype.

Key words: Logotype, image, discourse analysis, semiotics 


\section{Prefacio. Origen y objetivos del trabajo}

Al margen de su función de "marcaje" de productos, el logotipo condensa los valores de la compañía a la que representa, constituyendo su núcleo de significación, y el discurso que éste proyecta puede resultar decisivo en la construcción (no sólo "reflejo" o "transmisión") del valor diferencial de la compañía respecto a otras de la competencia (cfr.: Floch, 1993: 67-99; Semprini, 1995: 15, 19-24). Asimismo, con el tiempo el logotipo y el discurso que éste vehicula pueden pasar a formar parte de la cultura popular, de tal forma que el estudio de la significación de los logotipos se puede concebir como parte del estudio general sobre la construcción del imaginario social y el importante papel que juegan al respecto los medios de comunicación de masas y las grandes compañías comerciales.

En este contexto, este trabajo tiene como objetivo la definición de un modelo de análisis para el estudio de la imagen-logotipo como discurso visual, y su puesta a prueba a través del análisis del universo semántico proyectado por el logotipo de Adidas. El modelo de análisis que aquí se planteará está inspirado en un estudio previo de X. Ruiz Collantes acerca de la significación del imagotipo de Nike (Ruiz Collantes, 1999). Junto al estudio mencionado, las fuentes teóricas y metodológicas del trabajo son las semióticas icónica y plástica del signo visual (Groupe M, 1993), la semiótica narrativa y discursiva (Greimas, 1971; Courtés, 1980, 1997) y la teoría de análisis textual de la obra audiovisual de Casetti y Di Chio (1996), más algunos aspectos específicos de la Psicología de la Percepción Visual (Gombrich, 2004; Arnheim, 1979).

Respecto a la posible utilidad didáctica de este trabajo, cabe tener en cuenta que los logotipos, en tanto que discursos visuales complejos vehiculados a través de una mínima expresión, pueden resultar un objeto de estudio especialmente apropiado como aproximación inicial al análisis textual de la imagen.

El trabajo se divide en dos bloques fundamentales:

- Parte I: Definición de un modelo de análisis para el estudio de la imagen-logotipo como discurso visual

- Parte II: Estudio de caso: la significación de la marca Adidas como discurso visual

A partir de este punto se abordan cada uno de ellos.

\section{PARTE I. Definición del modelo de análisis}

Este trabajo se enmarca en el análisis textual de la imagen. La perspectiva del análisis textual de la imagen tiene como asunción teórica central la consideración de la imagen como vehículo de un espectro de significaciones que es posible generalizar y abstraer hacia una coherencia discursiva general, un "mensaje" articulado y coherente. 
A nivel metodológico, todo procedimiento de análisis discursivo parte de la "descomposición" del texto, prosigue con la tarea de análisis en sí misma, una posterior "recomposición" de los valores extraídos y, finalmente, desemboca en la "metacomprensión" o "comprensión del modo en que se comprende", finalidad última del análisis textual (Casetti y Di Chio, 1996: $17,23)$.

La "descomposición" del texto se basa en dos condiciones elementales de partida: la definición de uno o un conjunto de segmentos o unidades de referencia del análisis ("segmentación") y la delimitación de una serie de secciones de significación, transversales a dichas unidades ("estratificación") (cfr.: Casetti y Di Chio, 1996: 36).

Aunque, en un proyecto de análisis concreto, la segmentación y la estratificación dependen de factores "ad hoc" del propio proyecto, el propósito de construir un modelo general de análisis conduce a interpretar la segmentación y estratificación en su acepción más general En este sentido, la estratificación enlaza con la delimitación de códigos de significación relativamente universales o "lenguajes" (por ejemplo, el lenguaje plástico, el lenguaje audiovisual, etc.). Respecto a la segmentación del texto en sus "porciones" fundamentales, dicho procedimiento enlaza con la noción de esquemas discursivos, moldes sintácticos de organización de la información textual como la estructura de roles narrativos o la estructura enunciativa $^{1}$. En relación con la segmentación, un esquema o estructura discursiva canónica se puede concebir como un repertorio de unidades/segmentos de análisis universales.

\section{Estratos de significación de la imagen-logotipo: estrato plástico y estrato icónico}

Las características del logotipo lo presentan como un objeto visual que posee dos estratos de significación fundamentales: un estrato plástico y un estrato icónico².

Aunque posteriormente se irá matizando la distinción entre lo plástico y lo icónico, por el momento basta considerar el signo icónico como un signo visual con valor referencial más predicación (tal como recuerda la máxima semiótica, no es posible el "content" sin "comment") y el signo plástico como un signo visual con significación pero carente de referencialidad.

\subsection{Elementos de significación plástica}

Según la teoría del lenguaje plástico del Groupe M (1993: 167), los signos plásticos fundamentales son la forma, el color y la textura. Concretamente, los principales tipos de elementos plásticos y sus correspondientes parámetros de significación consisten en lo siguiente (Groupe M, 1993: 167 y ss.):

\footnotetext{
${ }^{1}$ Se puede encontrar una clara explicación sobre ambas perspectivas estructurales de análisis textual aplicadas al estudio de la obra audiovisual en: Casetti y Di Chio (1996).

2 Para mayor simplicidad, en este estudio se subsume el nivel tipográfico en el nivel plástico (esto significa que se consideran las letras como formas generales, sin atender a las convenciones expresivas más particulares del código tipográfico).
} 
La forma: definida por la articulación de los parámetros de ubicación, orientación y tamaño, más cualidades del contorno (rectilíneo vs. curvo, continuo vs. con irregularidades, etc.).

El color: definido por la articulación de los parámetros de tonalidad, saturación y claridad.

La textura: micro-topografía de la imagen definida por la articulación de dos parámetros: un tipo de "elemento repetido" y su "ley de repetición".

Cada uno de los elementos plásticos y sus parámetros correspondientes puede proyectar significación a través de un amplio espectro de ejes semánticos posibles, cuya actualización/selección en la interpretación del observador dependerá, fundamentalmente, de la interrelación de los diferentes elementos plásticos, y de los elementos plásticos con el conjunto de la imagen. Este proceso, comúnmente referido como la "influencia del contexto" sobre el significado de los signos (el "contexto" entendido aquí como contexto interno del enunciado), remite a la relevante cuestión de la búsqueda de la coherencia discursiva del texto. A modo de ejemplo, ejes semánticos generalmente atribuidos al color, como "frialdad vs. calor", "pureza vs. impureza" o "naturalidad vs. artificialidad" tenderán a cobrar relevancia en la interpretación de una imagen si uno o ambos polos semánticos de dichos ejes es sugerido simultáneamente desde "otra parte" de la imagen. De esta manera el observador, así como el analista, va desgranando (y simplificando) progresivamente los ejes semánticos fundamentales que están en juego en la imagen.

\subsection{Elementos de significación icónica}

La definición clásica del signo como representación es la de "algo que está en lugar de otra cosa", un "sustitutivo" (Zunzunegui, 2007: 58). En relación con ello, a diferencia de un signo plástico un signo icónico no sólo posee significación sino también valor referencial, en el sentido de que moviliza el reconocimiento de un objeto ${ }^{3}$. Dicho valor referencial emerge, según la teoría del Groupe M (1993: 120), de la siguiente manera: el signo icónico presenta un vínculo entre un significante y un referente (concebido como unidad cultural y no empírica) de un mundo real o imaginario, vínculo que se sustenta en un "tipo" o modelo general cuya evocación comparten significante y referente. Por ejemplo, por así decirlo, tanto el /dibujo de una silla/ como una /silla-objeto/ tienden a evocar el mismo "tipo" general de "silla".

Todo significante icónico presenta alguna propiedad concreta compartida con el objeto que evoca (p. ej. el color blanco respecto a la metáfora de los dientes como "perlas") y, al mismo tiempo, una o un conjunto de diferencias. Dicho de otra forma, el significante icónico "cambia y conserva a la vez" su referente (Groupe M, 1993: 160).

3 ... en particular, mediante una relación de semejanza relativa, que puede estar sustentada en semejanzas físicas entre el patrón estimular del significante y del referente (cuando éste se actualiza ante un observador de forma empírica) pero, sobre todo, se debe a una relación de "semejanza" como producto de nuestras convenciones culturales sobre "qué es" semejanza, "realismo" o "verosimilitud" (cfr.: Zunzunegui, 2007: 67-68). 
Respecto al estudio de los logotipos figurativos (o la eventual parte figurativa de un logotipo), cabe recordar que los elementos icónicos poseen, además del componente referencial, una significación propia. Por ejemplo, el famoso cocodrilo de Lacoste no deriva solamente en el reconocimiento del animal "cocodrilo", sino que, también, éste en sí mismo y en función del modo en que es "puesto en escena", pone de relieve determinadas connotaciones ("naturalidad", "solidez", "fuerza").

\section{Las perspectivas plástica e icónica respecto al análisis del texto visual}

La distinción entre signo plástico y signo icónico resulta diferente a la contraposición entre el análisis del texto visual como dimensión icónica (mundo figurativo) y como dimensión plástica (composición abstracta). Por el momento, basta con plantearnos una simple pregunta: ¿si la consideración de formas, colores y texturas (elementos plásticos) es pertinente para el estudio de la significación de entidades icónicas (personajes, objetos, escenarios, etc.), como es obvio, en qué nivel se debería situar esa parte del análisis: como parte del nivel plástico o del nivel icónico?... Será necesario dar un pequeño rodeo para aclarar esta duda.

\subsection{Sobre el texto visual como mundo figurativo}

Con independencia de si nos encontramos ante un texto de apariencia abstracta o figurativa, se puede considerar el análisis de la dimensión icónica como una perspectiva estructural, que remite al análisis de la imagen "como si" ésta evocara un mundo figurativo, tanto si se trata de una fotografía como de una pintura abstracta ${ }^{4}$. El principio clave a considerar aquí, para recordar la validez universal de esta perspectiva, sería que "en toda imagen abstracta se puede proyectar un elemento figurativo".

La consideración del texto como mundo figurativo implica la asunción fundamental de que éste delimita un determinado espacio-tiempo, "habitado" por entidades concretas reconocibles (personajes, objetos, escenarios, estados, acciones, sucesos) ${ }^{5}$. Esto da lugar a un grado mínimo de evocación de un mundo posible o imaginario, que puede ser aprehendido "de abajo arriba" (obras figurativas) o bien proyectado "de arriba abajo" por el observador (obras abstractas). De estas observaciones se puede deducir un repertorio básico de unidades/segmentos de análisis universales para el estudio del texto como mundo figurativo:

\footnotetext{
${ }^{4}$ Este enfoque teórico sigue una propuesta de Greimas y Courtès consistente en abordar la iconicidad no sólo como un problema de la teoría de los signos sino también como un problema discursivo. Acerca de esta cuestión: Zunzunegui, S. (2007: 71-72: "Para una reformulación del iconismo"): "... El concepto de iconicidad queda definitivamente desvinculado de sus lazos con unas específicas familias de signos, para situarse en el nivel de los procedimientos generales de la producción del sentido".

${ }^{5}$ El estudio del texto visual como mundo figurativo, según aquí se propone, resulta próximo al "nivel discursivo" en el análisis semio-narrativo del texto. No obstante, aquí se pone énfasis especialmente en la noción de mundo figurativo como molde sintáctico de unidades hábiles para la proyección icónica de los logotipos (cfr.: Greimas y Courtés, 2006: 194).
} 
Un "Entorno" del mundo figurado, formado como delimitación de un ámbito de espaciotiempo determinado.

Unos "Individuos" del mundo figurado, que responden a dos tipos fundamentales:

Existentes: personajes, objetos, escenarios

Eventos: acciones, sucesos y estados ${ }^{6}$

Desde este enfoque, se entiende que las formas aparentemente abstractas pueden delimitar un determinado campo de proyección icónica; por ejemplo, el símbolo de Nike proyectado como estela en el aire de un movimiento ágil y ascendente (Ruiz Collantes, 1999). No por menos obvia que en los imagotipos directamente figurativos, la dimensión icónica deja de formar parte de la discursividad de los imagotipos abstractos.

El ejercicio de proyección icónica, si se quiere junto a la consideración del ámbito comercial de la marca en términos generales, puede ser muy útil en el análisis de logotipos abstractos, ya que proporciona al analista un "marco" interpretativo concreto, a partir del cual se podrán precisar mejor los valores derivados del análisis plástico. Así, por ejemplo, una composición plástica muy estilizada podría remitir a "elegancia" o a "agilidad", pero en relación con un ámbito comercial relativo al deporte y/o a una proyección icónica relativa a una acción de movimiento físico, "agilidad" sería el valor que cabría poner más de relieve respecto al discurso de fondo de la imagen (cfr.: Ruiz Collantes, 1999).

\subsection{Sobre el texto visual como composición abstracta}

Respecto a la dimensión plástica de la imagen como perspectiva global de análisis textual, con independencia de si nos encontramos ante una imagen altamente figurativa o más bien abstracta, el análisis del texto visual como composición plástica funciona como una interpretación de la imagen en clave abstracta. El principio fundamental a recordar en este caso, para la validez universal de la perspectiva, sería que "en toda imagen figurativa anida una imagen plástica". Este enfoque del análisis invoca una espacialidad de la imagen tomada como bidimensional, y un espacio cerrado por un marco en el que "se ubican formas que no remiten a seres [concretos] que habitan un mundo real o imaginario, sino que son formas con valor en sí mismas" (Ruiz Collantes, 1998).

Existen diferentes teorías para el estudio de la composición abstracta de la imagen, pero se pueden destacar las de Gombrich (2004) y Arnheim (1979), basadas en el estudio de dinámicas esenciales en la percepción visual. Según Gombrich (2004), la percepción de la

\footnotetext{
${ }^{6}$ La distinción entre los sub-tipos de individuos como "existentes" y "eventos" se adapta de: Chatman (1990: 19). Por otro lado, a partir de una teoría de L. Dolezel (cfr.: 1999: 92) se adopta el siguiente criterio de distinción entre acciones y sucesos: las acciones se pueden concebir como hechos producidos por agentes que poseen intencionalidad (sujetos humanos, animales, personajes de mundos de fantasía) y los sucesos como hechos producidos por agentes carentes de intencionalidad propia (fenómenos naturales, elementos sobrenaturales, tal vez el azar, etc.).
} 
composición abstracta global de una imagen se fundamenta en una búsqueda espontánea de "orden" por parte del sistema visual, relativamente independiente de la función de reconocimiento de las formas (iconicidad). En este sentido, el valor expresivo de la composición visual se basaría en una dialéctica entre el "orden" y su contraprincipio activo, el "desorden" o "variedad"7. Dentro del marco general de la dialéctica entre orden vs. desorden compositivo se puede integrar también la consideración del "equilibrio compositivo", en tanto que búsqueda perceptiva de un tipo de orden fundamentado en la relación entre los "pesos visuales" de la composición plástica (Arnheim, 1979: 25).

Según X. Ruiz Collantes (1998), el análisis de la composición abstracta de la forma basado en la dialéctica de orden/redundancia vs. desorden/variabilidad remite a una "dimensión informativa" de la forma, que podría ser complementada con otras dimensiones semánticas, fundamentalmente la "dimensión objetual" y la "dimensión espacial".

La "dimensión objetual" se basaría en una proyección genérica o poco concreta de la imagen (o de una forma componente de la imagen) como objeto/sujeto físico, lo cual lleva a identificar decantaciones semánticas de la forma respecto a ejes como "estabilidad vs. inestabilidad", "equilibrio vs. desequilibrio", "estatismo vs. dinamismo", "pesado vs. ligero", fuerte vs. frágil", etc.

La "dimensión espacial" se basa en una proyección no-concreta del fondo de la imagen como espacio físico, lo cual permite atisbar decantaciones semánticas de las formas respecto a ejes como "etéreo vs. terrenal", "fantasía vs. realidad", "central vs. marginal", "detrás vs. delante", etc.

El matiz de la proyección objetual o espacial de la imagen de forma "genérica" o "noconcreta" es importante aquí, por el motivo de que una proyección de mayor concreción ( $p$. ej., la forma no sólo como "objeto/sujeto" en general sino como "martillo", "escalera" o "ave") nos llevaría ya al análisis de la composición desde la perspectiva icónica (la imagen como mundo figurativo). En cambio, este tipo de proyecciones icónicas difusas o particularmente genéricas se pueden emplazar dentro del análisis de la imagen como composición abstracta.

\section{Un modelo de análisis para el estudio de la imagen-logotipo como discurso visual}

La combinación de un determinado tipo de organización sintáctica del texto ("segmentación") con una determinada delimitación de secciones de análisis ("estratificación") permite definir un modelo de análisis para el estudio del discurso.

\footnotetext{
${ }^{7}$ La teoría de Gombrich invita a su vinculación con la Teoría de la Información, en relación con la contraposición entre los principios de "simplicidad" (orden, previsibilidad) e "información" (desorden, imprevisibilidad).
} 
En el caso que nos ocupa, tras la definición de dos estratos pertinentes de análisis (nivel del signo plástico y nivel del signo icónico), y de dos perspectivas de organización del discurso (el texto visual como composición abstracta y el texto visual como mundo figurativo), obtenemos un esbozo provisional de modelo de análisis, que puede ser representado de la siguiente manera:

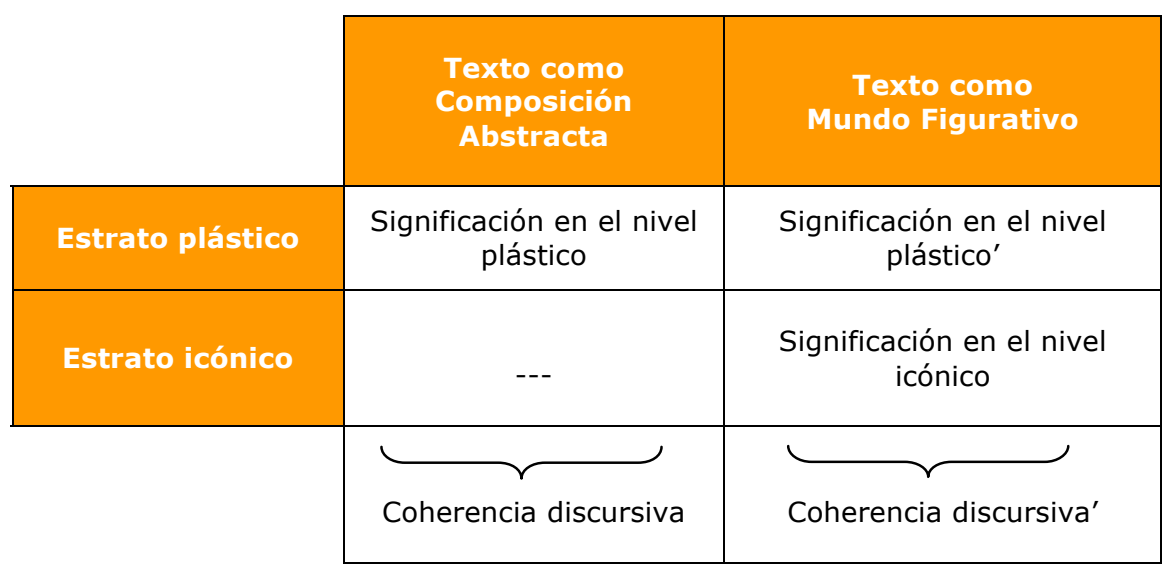

Se deben establecer tres consideraciones sobre este esquema todavía provisional:

1.- En primer lugar, evidentemente en el estudio del texto visual como composición abstracta no procede un nivel de análisis de signos icónicos. A la composición plástica general se llega a través de la omisión de la iconicidad, dejándola al margen en la organización global de la información visual, y por tanto reduciendo la imagen a su existencia como articulación de formas, colores y texturas.

2.- Por otro lado, podemos observar que, finalmente, hemos alcanzado una respuesta clara para aquella duda inicial: "¿si la consideración de formas, colores y texturas es pertinente para el estudio de la significación de entidades icónicas (personajes, objetos, escenarios, etc.), como es obvio, dónde se debería situar esta parte del análisis: como parte del nivel plástico o del nivel icónico?". En este sentido, el esquema plantea un espacio de análisis claramente delimitado para el signo plástico como "parte de" una dimensión icónica del texto visual (del texto visual como mundo figurativo) (cuadrante superior derecho respecto a las cuatro celdas interiores del esquema).

3.- Este esquema provisional posee un carácter modular, en el sentido de que daría lugar, en la fase final del análisis, a dos "coherencias discursivas" del texto desde diferentes enfoques, y no explicita de momento un eslabón de unificación y abstracción final hacia la "coherencia discursiva general" del texto. Concretamente, los dos módulos de análisis textual de la imagen que plantea el esquema son producto de las siguientes combinaciones:

Módulo 1: texto visual como composición abstracta + estrato plástico;

Módulo 2: texto visual como mundo figurativo + estratos plástico e icónico. 
El análisis de la coherencia discursiva global del texto resulta necesario, y puede abordarse de dos formas fundamentales: por un lado, mediante una elaboración de "conclusiones" finales sobre el discurso de la imagen donde el analista asuma la tarea de sintetizar y buscar coherencia general respecto a la información de ambos módulos; por otro lado, mediante una previa "modelización final", a través de algún tipo de estructura discursiva sobre la que se unifique la información semántica de ambos módulos. En este sentido, en este trabajo se van a proponer dos esquemas, diferentes y compatibles, para la ulterior modelización discursiva en la tarea de análisis del logotipo: (a) la estructura narrativa canónica, tal como propone Ruiz Collantes (1999), y (b) la estructura de relaciones simbólicas Sujeto vs. Entorno.

a) La estructura discursiva de roles actanciales, definida por A. J. Greimas (1971), constituye un esquema canónico para la construcción y comprensión de diversos tipos de textos "como si" fueran narraciones, independientemente de si se trata de películas de ficción, espots electorales, imagotipos de marcas deportivas, etc. La estructura greimasiana presenta tres ejes de narratividad fundamentales, que establecen seis unidades de referencia básicas, conocidas como "roles narrativos", más las "acciones/transformaciones" realizadas por cada uno de los actantes:

Eje Sujeto - Objeto: donde el "Sujeto" es un agente que realiza "acciones/transformaciones" para la consecución de un determinado objetivo, el "Objeto". Se suele considerar al Sujeto en clave de "héroe" de la narración.

Eje Destinador - Destinatario: donde el "Destinador" es un agente que plantea o prescribe la búsqueda de un "Objeto" a un determinado "Destinatario".

Eje Adyuvante - Oponente: donde el "Adyuvante" es un agente benefactor para los intereses del Sujeto en su intento de consecución del Objeto y, a la inversa, el "Oponente" es un agente perjudicial o un obstáculo para el Sujeto en su intento de consecución del objetivo.

b) Respecto a la posible utilidad del eje Sujeto vs. Entorno para el análisis discursivo de la imagen, cabe retrotraernos a un aspecto elemental de la Psicología de la Percepción Visual: la segregación de figura vs. fondo (estudiada originalmente por E. Rubin y desarrollada después por la Escuela de la Gestalt) (ver, p. ej.: Luna y Tudela, 2006: 225-230).

Las relaciones figura/fondo resultan esenciales en el estudio de la percepción visual, pero deben ser ligeramente reinterpretadas en cuanto a su potencial como eje discursivo, más allá de su "superficie" perceptiva.

En este sentido, la interpretación del eje figura/fondo en clave de relaciones simbólicas sujeto/entorno abre un espacio de análisis discursivo que puede resultar muy interesante para comprender la significación de la imagen publicitaria y de la imagen en general. 
Para ello, es conveniente tener en cuenta que el análisis de relaciones simbólicas sujeto/entorno no debe aplicarse de forma "mimética" o necesariamente "literal" respecto al correlato perceptivo de figura/fondo. Por ejemplo, en algunos textos visuales el Sujeto se "extiende" como figura y fondo al mismo tiempo (a veces para expresar la omnipresencia del "mal", como ocurre en algunos carteles de cine de terror o de gángsters), en otras imágenes se juega con la "inversión" del Sujeto como fondo y el Entorno como figura, etc. ${ }^{8}$

En conclusión, si, partiendo del esquema anterior, engastamos la Estructura Narrativa y el eje Sujeto/Entorno como eslabones ulteriores del proceso analítico (escogiendo un modelo o ambos, de forma complementaria), obtenemos el siguiente esquema resultante:

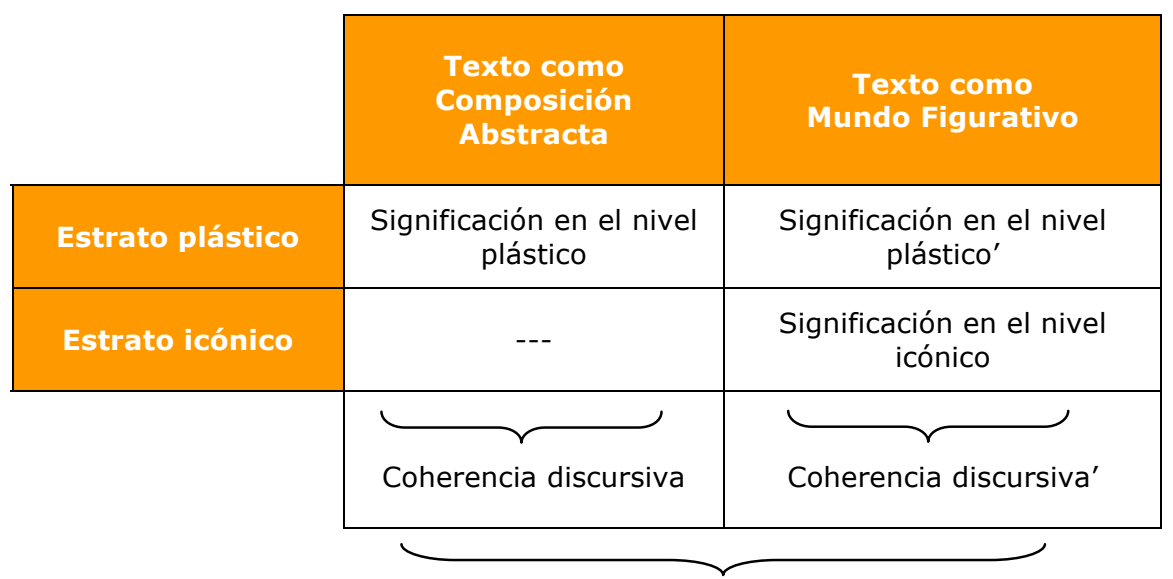

Texto como Estructura Narrativa / como Relación simbólica Sujeto/Entorno

Coherencia discursiva general

Una vez completada la definición del modelo de análisis para el estudio del logotipo como discurso visual, se aborda ahora la segunda parte del trabajo: la aplicación del modelo al análisis del discurso proyectado por el logotipo

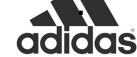

\footnotetext{
8 En relación con esto mismo, en una obra visual secuencial, aunque todo plano posea relaciones figura/fondo individualmente, la relación verdaderamente significativa para el análisis discursivo podría consistir en el plano " $A$ " como imagen del Sujeto y el plano " $B$ " (consiguiente) como imagen del Entorno, contrastadas mediante el montaje. Por ejemplo, un plano " $\mathrm{A}$ " donde el protagonista y un escenario interior (p. ej., su habitación) contribuyen conjuntamente a sugerir la personalidad o estado anímico del personaje (Sujeto), y en el plano siguiente ("B") se muestra un espacio exterior (p. ej., ciudad tumultuosa), que constituiría ya el Entorno al que se confronta, discursivamente, el protagonista.
} 


\section{PARTE II. Estudio de caso: la significación de la marca Adidas como discurso visual}

\section{Análisis del texto visual como Composición Abstracta}

Desde el enfoque del texto visual como composición abstracta, el logotipo de Adidas debe ser considerado como una forma bidimensional abstracta que no remite a un referente externo concreto y perteneciente a un mundo real o posible (Ruiz Collantes, 1999). En este módulo de análisis de textualidad visual se deben tomar en cuenta, como estratos de análisis de la significación, los principales elementos plásticos: forma, color y textura, y sus parámetros fundamentales (ver la primera parte de este estudio).

En este caso, se prestará especial atención a la configuración de la forma (al margen del cromatismo y la textura) y, en cuanto a su valor semántico, nos centraremos fundamentalmente en la "dimensión informativa".

El logotipo de Adidas se basa en una forma global ("gestalt") próxima al triángulo equilátero. El triángulo es una forma geométrica básica, lo cual aporta un tono de "simplicidad" y "esencialidad" a la composición. Por otro lado, el triángulo posee una base muy estable pero al mismo tiempo apunta hacia arriba, hacia un despegue de la superficie ilusoria bajo la base. Esto sitúa al triángulo como una forma geométrica intermedia entre la máxima "estabilidad" o "terrenalidad" del cuadrado y la cualidad "etérea" y "trascendente" del círculo. Así, como "gestalt" simbólica, el triángulo atesora un balance entre lo "terrenal" y lo "etéreo", entre la "estabilidad" y lo "lúdico". Un análisis micro-estructural de los rasgos particulares del triángulo segmentado de Adidas redundará considerablemente en este posicionamiento de "equilibrio" simbólico.

Tal como se había comentado anteriormente, la percepción de la composición abstracta de una imagen tiene como base la búsqueda espontánea de "orden" por parte del sistema visual (Gombrich, 2004), relativamente independiente de la función de reconocimiento de las formas (iconicidad). En este sentido, el valor expresivo de la composición visual se basa en una dialéctica entre el "orden" o "regularidad" y su contraprincipio activo, el "desorden" o "variedad". El "orden" se suele asociar a valores como "estabilidad", "seguridad" o "terrenalidad", etc., mientras el "desorden" se asocia a valores contrapuestos como "inestabilidad", "imprevisibilidad" o "diversión", etc. En el triángulo segmentado de Adidas encontramos "orden" y "variedad" en cantidades muy equilibradas:

En primer lugar, encontramos "orden" en factores plásticos como la rectangularidad de las formas componentes del triángulo, su igualdad de anchura, la repetición de ángulos rectos, y el ritmo regularizado en la yuxtaposición de las barras: la longitud de las barras presenta variación por incremento, pero de forma proporcionada. 
Por otro lado, la presencia de la tipografía "adidas" en la parte inferior y con orientación horizontal ejerce como una "base" metafórica de la forma triangular, que viene a subrayar su "estabilidad".

Además, el triángulo segmentado de Adidas está definido por contornos rectilíneos en lugar de curvos.

Este conjunto de propiedades de la "gestalt" remite, a nivel micro-estructural, a valores como la "estabilidad", la "seguridad" o la "sobriedad", por contraposición al "dinamismo", la "imprevisibilidad" o lo "lúdico".

Una forma cuya proyección semántica se base con cierta exclusividad en valores como la "estabilidad", "sobriedad" y derivados puede activar también valores asociados de tono generalmente negativo, como "aburrimiento" o "pesadez", etc. Se puede establecer la consideración de que, por ejemplo, la marca de un banco, de una caja de ahorros o de una compañía de seguros podría asumir en mayor grado dicho "riesgo" de ambivalencia semántica, pero, respecto a una marca deportiva, por lo general este tipo de valores se suelen matizar de alguna manera, hacia connotaciones de mayor tensión y dinamismo. El imagotipo de Adidas es un claro ejemplo al respecto.

En este sentido, una propiedad plástica del imagotipo de Adidas muy diferente a las anteriores, emerge con especial relevancia: la leve asimetría de la "gestalt" triangular.

El imagotipo de Adidas conforma una "gestalt" próxima al triángulo equilátero pero asimétrica: el vértice superior no corresponde, en su perpendicular con la base, a un punto equidistante entre los dos vértices inferiores. Se trata de una ligera asimetría, que mantiene implícita la referencia de una "gestalt" simétrica. Este tipo de fenómeno perceptivo, donde una forma ligeramente desviada de un patrón simple de referencia se distingue levemente de éste, pero manteniendo su evocación implícita, es el origen de la "tensión perceptiva" de las formas. Uno de los valores fundamentales que proyecta la "tensión perceptiva" de las formas es el valor de "dinamismo" (Arnheim, 1979: 430) ${ }^{9}$.

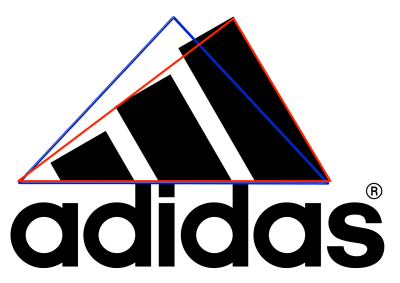

Se puede apreciar también otro detalle de asimetría en la composición de la imagen: la asimetría entre la existencia de un vértice cerrado en la parte inferior derecha del triángulo

\footnotetext{
${ }^{9}$ Asimismo, en relación con la tensión perceptiva cabría añadir que la altura del triángulo implícito al que remite el imagotipo es cercana pero ligeramente inferior a la necesaria para reproducir el modelo canónico de triángulo equilátero.
} 
gestáltico, y su ausencia (vértice "cortado") en el lado inferior izquierdo. De nuevo, este detalle de sutil asimetría aporta tensión perceptiva a la composición.

La conexión entre los valores de "tensión" y "dinamismo" se refuerza a través de otros aspectos de la composición del logotipo: especialmente, la orientación diagonal de las tres barras que componen el triángulo gestáltico, junto a las diagonales virtuales del perímetro que forman los contornos implícitos del triángulo. A diferencia de las líneas en horizontal/vertical, las líneas compositivas diagonales proyectan fundamentalmente valores como "dinamismo", "tensión", "ligereza", etc.

Por otro lado, se puede apreciar que la superficie del triángulo gestáltico no es una superficie continua y unitaria, sino discontinua, fragmentada y con variaciones internas. Discontinua y fragmentada por la yuxtaposición de tres barras componentes de la gestalt triangular, además de la relación de "desencaje" entre los lados superiores de las barras, y con variaciones por el incremento de longitud de las barras. Las discontinuidades, la fragmentación y las variaciones aportan cierto grado de "desorden" y "dinamismo" al conjunto de la composición.

Finalmente, la forma redondeada de las letras y su delgadez en la tipografía de "adidas" contribuye a aportar "ligereza" al conjunto de la composición, balanceando el propio valor de "estabilidad" que proyectan la ubicación inferior y la orientación horizontal del bloque tipográfico.

En resumen, el cuidado balance de nivel macro-estructural que presenta el esquema en triángulo, como arquetipo de equidistancia entre la "terrenalidad" y la "sobriedad" del cuadrado y lo "etéreo" o "lúdico" del círculo, se reproduce también a nivel micro-estructural, por la interacción entre dos focos contrapuestos de configuración plástica: por un lado, propiedades plásticas como el orden/regularidad de los componentes, la rectilineidad, la compacidad y la ubicación inferior con horizontalidad de la tipografía ("estabilidad", "seguridad", "terrenalidad") y, por otro, las leves asimetrías, las líneas diagonales, las discontinuidades, la fragmentación y las variaciones, más la forma redondeada y la delgadez de las letras en la tipografía ("dinamismo", "tensión", "ligereza").

Restaría añadir una breve consideración respecto a los elementos plásticos del color y la textura. En primer lugar, el caso tomado para este estudio es un logotipo en blanco y negro, pero a menudo los logotipos cambian de color según el lugar que ocupan en los distintos vehículos publicitarios donde se encuentran. En cuanto a la textura, aunque a priori podría parecer significativa su ausencia en la imagen, lo cierto es que en los logotipos raramente se encuentran valores texturales de la imagen. Por tanto, en este caso parece poco adecuado aventurarnos a matizar las significaciones de la forma en función de las significaciones del color y la (ausencia de) textura. 
Como conclusión preliminar en este módulo de análisis, se puede observar que el discurso visual de Adidas proyecta un universo semántico centrado en un sutil pero muy calculado equilibrio entre "estabilidad" y "dinamismo", "seguridad" y "tensión", "terrenalidad" y "ligereza", lo "sobrio" y lo "lúdico". Así, de forma provisional, podemos interpretar el discurso general de la marca Adidas como la prescripción de equilibrio entre este tipo de extremos de la experiencia vital. Previsiblemente, el resto de módulos de análisis contribuirán a matizar y concretar estas observaciones.

\section{Análisis del texto visual como Mundo Figurativo}

En el estudio del texto visual como mundo figurativo, la imagen-logotipo se proyecta como representación de un objeto/sujeto concreto que existe en un mundo real o posible, y que forma parte de un entorno, dentro de un espacio tridimensional y un tiempo determinados (Ruiz Collantes, 1999). A diferencia del módulo anterior, en el módulo de análisis del texto visual como mundo figurativo se aúnan dos estratos distintos de significación: el estrato plástico y el estrato icónico (ver la primera parte del estudio). Empezaremos por la proyección semántica desde el nivel icónico, para matizar después los valores identificados a través de los elementos plásticos como rasgos del plano de expresión de figuras icónicas.

La elevada abstracción del imagotipo de Adidas exige una lectura icónica proyectada "de arriba abajo" por el observador. No obstante, la forma del imagotipo no remite, en términos pragmáticos, a una deriva infinita de posibilidades icónicas; es decir, ciertas proyecciones icónicas emergen de forma "natural" y "prioritaria", por encima de otras.

Por ejemplo, la forma semi-triangular del imagotipo activa con especial facilidad su interpretación icónica como "montaña" o "pirámide". A su vez, la yuxtaposición en progresiva ascensión de los segmentos del lado izquierdo sugiere la visualización de esa parte del imagotipo como "escalera". Entre ambos casos, emerge la idea común de "ascensión" como valor fundamental, y la actividad metafórica de "escalar una montaña". Esta actividad proyectada connota la "ascensión", el "éxito", como algo que requiere de un esfuerzo con "constancia" y de una suma de pequeñas mejoras, paso a paso, día a día...

Por otro lado, la concepción visual de las barras componentes del triángulo gestáltico como rectángulos incompletos remite a la interpretación general de la imagen como un conjunto de objetos alargados que se "hunden" en sentido oblicuo en una determinada superficie, y/o que están siendo "inclinados" por alguna fuerza natural externa. Esta clave interpretativa puede dar lugar a variadas posibilidades icónicas: "estacas torcidas", "torres inclinadas", "árboles inclinados por el viento", "mástiles semihundidos", etc. A pesar de la heterogeneidad posible en este caso, se puede observar un rasgo común a este espectro semántico: la idea de que, en el mundo figurativo proyectado, existe una fuerza externa (el paso del tiempo, fenómenos naturales, la fuerza del viento, etc.) que afecta de forma sustancial a las entidades que habitan dicho mundo figurado. 
Por último, la yuxtaposición de las barras diagonales en un ritmo creciente pero perfectamente proporcionado puede remitir a la figura de un "medidor tecnológico" que forma parte de algún aparato o máquina (p. ej., el control de volumen de una cadena musical). Desde este punto de vista, el ritmo creciente de las barras de Adidas alude a la interacción de dos valores fundamentales: el "cambio/aumento" con "control". Esto se podría vincular a las acciones de los individuos del mundo figurativo: en dicho mundo figurativo, la actividad orientada a "cambiar" o "mejorar" las cosas remite a acciones asociadas a la "potencia con control" por parte del sujeto.

Finalmente, respecto a los "personajes" del mundo figurativo, se debe subrayar precisamente su ausencia en la proyección icónica. Las barras diagonales del imagotipo remiten, metafóricamente, a elementos del mundo natural ("árboles") o del mundo cultural ("estacas", "torres", "medidores tecnológicos"), pero no a sujetos con iniciativa e intencionalidad propias (personajes). El mundo figurativo es, por tanto, un mundo "vacío", "a la espera de personajes", sobre todo a la espera de un "héroe". Este "héroe" es, implícitamente, el comprador de Adidas.

Respecto a los rasgos plásticos de estos elementos icónicos proyectados, se pueden observar ciertos matices interesantes, algunos de ellos retomados del apartado anterior: por ejemplo, la ligera asimetría del triángulo gestáltico aporta "tensión" a la "montaña" figurada, y sugiere así el movimiento de "ascensión" como ascensión con "esfuerzo" y cierta "imprevisibilidad" del resultado (sentido de "aventura"). A su vez, la repetición de segmentos de la misma medida e igual orientación en el lado izquierdo del triángulo atribuye "tesón" y "perseverancia" a la actividad de ascensión. La regularidad plástica de las barras diagonales, mediante la proporción en su ritmo de aumento, asigna también el valor de "control" a la actividad de ascensión.

Por otro lado, la firmeza de la base del triángulo, remarcada por la ubicación inferior y la horizontalidad de la tipografía "adidas", añade "solidez" e "imperturbabilidad" al mundo figurado, un mundo que "no cambia" o que "se resiste al cambio".

Finalmente, la rectangularidad, anchura y peso visual de los bloques diagonales que componen el triángulo acentúan de forma reseñable la "fuerza" de las condiciones externas del mundo figurativo: sólo unas fuerzas externas poderosas podrían "doblegar" (afectar) a elementos aparentemente tan compactos.

\section{Coherencia discursiva general. El texto visual como Estructura Narrativa / como Relación simbólica Sujeto/Entorno}

Tal como se había planteado en nuestro modelo de análisis, tras la extracción de los valores fundamentales en los módulos del texto como composición abstracta y del texto como dimensión figurativa, puede ser conveniente subsumir ambos universos semánticos en una estructura integradora, que nos ayude a alcanzar una visión global sobre los aspectos 
esenciales del discurso visual que proyecta la imagen-logotipo (ver la primera parte del estudio).

En este estudio, realizaremos esta operación de dos formas diferentes, pero compatibles. En primer lugar, tomando la estructura narrativa como referencia para la modelización final y, en segundo lugar, tomando el eje de relaciones simbólicas sujeto/entorno como referencia para la modelización final.

\subsection{El texto visual como Estructura Narrativa.}

Desde un punto de vista narrativo, el mundo evocado por el logotipo de Adidas es un mundo "a la espera de un héroe" -el comprador de Adidas- (Sujeto) que, si quiere "ascender" o "mejorar" (Objeto), deberá superar unas duras condiciones externas del mundo: "montaña empinada", "viento"... (Oponente), actuando con "perseverancia" y "rebeldía" pero también con "control" (acciones/transformaciones del Sujeto).

Ahora bien, a estas alturas del análisis deberíamos integrar también con lo anterior los valores fundamentales del texto como composición plástica. Es decir, debemos preguntarnos qué papel juega, en la estructura narrativa, el universo semántico proyectado por la composición abstracta de la imagen.

A este respecto, habíamos comprobado que, plásticamente, el discurso visual de Adidas proyecta un universo semántico centrado en un mesurado equilibrio entre "estabilidad" y "dinamismo", "seguridad" y "tensión", "terrenalidad" y "ligereza", etc. La connotación de "equilibrio" complejo entre extremos de la experiencia vital enlaza sobre todo, respecto al módulo de análisis icónico, con el valor del "control". En última instancia, podemos integrar los valores del "equilibrio" y el "control" como rasgos semánticos vinculados a las acciones/transformaciones que el relato prescribe al Sujeto destinado a "cambiar las cosas" en el mundo narrativo (el héroe / comprador de Adidas).

De este modo, el "héroe" narrativo de Adidas queda perfilado como un tipo de héroe "fuerte", "dinámico" y "valiente/rebelde", pero también "equilibrado", en gran medida "realista".

\subsection{El texto visual como Relación simbólica Sujeto/Entorno}

La última observación nos lleva ya, prácticamente de forma natural, a dedicar una parte final del análisis a la examinación del discurso que vehicula el logotipo en función del tipo de relaciones Sujeto-Entorno contenidas implícitamente en la imagen.

Desde este enfoque, se observa que, ante la relevante cuestión de "cómo cambiar y mejorar las cosas" de su Entorno, el Sujeto podría proyectarse como un individuo capaz de trascender completamente las condiciones externas, o bien como un sujeto que, más que 
imponer su voluntad en el entorno o evadirse del mismo, aprende a afrontar las circunstancias y a establecer un equilibrio entre el yo y las condiciones exteriores.

A juzgar por nuestro análisis, a este segundo tipo de Sujeto es al que remite la imagenlogotipo de Adidas: no a un Sujeto que impone su voluntad al Wntorno o logra una total liberación respecto a las condiciones externas, sino como un Sujeto capaz de armonizar el yo con las condiciones exteriores.

En conclusión, el discurso implícito de la imagen-logotipo de Adidas expondría un entorno (montaña) donde el sujeto/héroe, en lugar de eliminar totalmente o esquivar las dificultades/adversidades, prefiere afrontarlas, aprender, adaptarse y seguir avanzando paso a paso. De esta manera y no de otra intenta alcanzar sus objetivos: "ascender", "mejorar", "llegar más lejos"...

3.3. Consideraciones finales. Sobre el contraste discursivo entre la imagen-logotipo de Nike y la imagen-logotipo de Adidas

Las estrategias de posicionamiento de las marcas conllevan una distinción respecto a la competencia y, a nivel semántico, esto suele derivar en sustanciales contrastes discursivos entre los logotipos de diversas marcas. Un claro ejemplo al respecto es el contraste discursivo entre el universo semántico proyectado por el imagotipo de Nike, analizado por Ruiz Collantes (1999) y el universo semántico del logotipo de Adidas: , que aquí hemos analizado.

Según el análisis de Ruiz Collantes (1999), el discurso visual proyectado por el imagotipo de Nike se origina en una estela de movimiento que el Sujeto ha dejado en el aire tras de sí, al pasar fugazmente ante los ojos del observador. Dicha estela de movimiento remite a valores fundamentales como la "liberación" y la "trascendencia", ya que para tomar impulso el Sujeto prácticamente no ha necesitado siquiera apoyarse en el suelo (no hay rastro de superficie terrenal en la imagen de Nike); se trata de un movimiento extremadamente ágil y ligero, que connota la total emancipación del Sujeto respecto a las ataduras del mundo exterior. El Sujeto de la imagen de Nike es, por tanto, un Sujeto capaz de trascender las condiciones del mundo que le rodea, casi un "superhéroe".

El Sujeto promovido por la imagen de Adidas es, como hemos visto, un individuo bastante distinto. También busca, como el Sujeto de Nike, un objetivo de superación: "ascender", "Ilegar más lejos", pero no lo hace "suspendido en el aire", sino "escalando una montaña".

El héroe de Nike es un héroe caracterizado por su liberación de las condiciones exteriores, que puede y quiere escapar del mundo real, demostrar su total autonomía e independencia. En cambio, el héroe de Adidas es un héroe más "realista", caracterizado por su perseverancia y su capacidad de adaptación, un héroe que prefiere "pisar con los pies en la tierra", que no pretende escapar más allá de su realidad sino encararla de frente y llegar lejos, muy alto, pero paso a paso, día a día. 


\section{REFERENCIAS BIBLIOGRÁFICAS}

ARNHEIM, Rudolf (1979): Arte y percepción visual. Madrid, Alianza.

ARNHEIM, Rudolf (2001): El poder del centro. Madrid, Akal.

CASETti, F. y Di Chio, F. (1996): Cómo analizar un film. Barcelona, Paidós.

Chatman, S. (1990): Historia y discurso: la estructura narrativa en la novela y en el cine. Madrid, Taurus.

COURTÉS, Joseph (1980): Introducción a la semiótica narrativa y discursiva. Buenos Aires, Hachette.

COURTÉS, Joseph (1997): Análisis Semiótico del Discurso: del enunciado a la enunciación. Madrid, Gredos.

DOLEZEL, Lubomir (1999): Heterocósmica: ficción y mundos posibles. Madrid, Arco.

DondIS, Donis A. (1990): La sintaxis de la imagen. Barcelona, Gustavo Gili.

Eco, Umberto (1981): Lector in fabula. Barcelona, Lumen.

FLOCH, Jean-Marie (1993): Semiótica, marketing y comunicación. Barcelona, Paidós.

GOMBRICH, Ernst H. (1998): Arte e ilusión. Madrid, Debate.

GoMbrich, Ernst H. (2004): El sentido del orden. Madrid, Debate.

Greimas, Algirdas J. (1971): Semántica estructural. Madrid, Gredos.

Greimas, A. J. y COURTÉS, J. (2006): Semiótica: Diccionario razonado de la Teoría del Lenguaje. Madrid, Gredos.

GRouPE M. (1993): Tratado del signo visual. Madrid, Cátedra.

LunA, D. y TudelA, P. (2006): Percepción visual. Madrid, Trotta.

PARRAMón, José M. (1965): Así se compone un cuadro. Barcelona, Instituto Parramón.

Ruiz Collantes, Xavier (1998): Hacia un heurístico para la definición de los valores compositivos de la forma en la publicidad gráfica. Investigación no publicada. Barcelona, Universitat Pompeu Fabra.

Ruiz Collantes, Xavier (1999): "El significado de la marca Nike", en Formats, no 2. Barcelona, Universitat Pompeu Fabra. On-line en http://www.iua.upf.es/ formats/formats2/rui_e.htm (consultado: 01/09/2009).

SEMPRINI, Andrea (1995): El marketing de la marca. Una aproximación semiótica. Barcelona, Paidós.

van DiJk, Teun A. (1993): Texto y contexto. Semántica y pragmática del discurso. Madrid, Cátedra.

Zunzunegui, Santos (2007): Pensar la imagen. Madrid, Cátedra.

[Recibido: 13 de octubre de 2010. Aceptado (sin cambios): 19 de septiembre de 2011.] 